\title{
Une méthodologie multidisciplinaire pour l'étude du littoral : Cas du littoral de Mohammedia (Maroc)
}

\author{
F. Gouaud ${ }^{1}$, A. Hourimeche ${ }^{2}$, V. Rey ${ }^{1}$, M. Chagdali ${ }^{2}$ et M. Idrissi ${ }^{1,2}$ \\ ${ }^{(1)}$ LSEET-LEPI, Université du Sud Toulon-Var, B.P 20132 F 83957, la \\ Garde, Cedex, France \\ ${ }^{(2)}$ LCSM - LGSE, Faculté des Sciences Ben M'sik, BP 7955, Sidi \\ Othmane, Université Hassan II - Mohammedia, Casablanca, Maroc
}

\section{Résumé}

Le littoral a fait l'objet d'un certain nombre d'aménagements côtiers qui modifient la répartition et la distribution des sédiments, susceptibles de conduire à la dégradation de l'environnement naturel. Pour caractériser l'évolution d'un littoral, on présente une méthodologie basée sur deux approches complémentaires : d'une part une étude descriptive à partir de mesures in situ de granulométrie, de minéralogie, de photographies aériennes et d'autre part une modélisation numérique de la houle à la côte, et de l'évolution du trait de côte. Cette méthodologie est appliquée à l'étude du littoral de Mohammedia (Maroc) dont les aménagements portuaires ont fortement influencé l'évolution du trait de côte à long terme.

\section{Mots Clés}

Trait de côte, transport sédimentaire, évolution à long terme

\section{$\underline{\text { Abstract }}$}

Coastal management may lead to sediment transport changes including grain sorting, and beach erosion. A methodology based on two complementary approaches is presented for the characterization of shoreline evolution. The first is based on field measurements, sediment property analyses and aerial photographs, the second on numerical approach for wave transformation in the nearshore and shore evolution. This methodology is applied to the Mohammedia coastal zone, which long term dynamics are strongly affected by the Mohammedia harbour infrastructures.

\section{Introduction}

Le littoral est un domaine privilégié pour étudier et mieux comprendre l'incidence des processus naturels et anthropiques sur la dynamique sédimentaire actuelle. 
La morphologie du domaine d'étude, le littoral de Mohammedia (Maroc), est caractérisée par l'alternance des plages sableuses et des plages à platiers rocheux. A l'Ouest, au niveau du cap de Fedala, la pente du fond est importante. Une dorsale sous marine (D1) se trouve dans le prolongement du cap. La grande digue du port de Mohammedia relie la pointe du cap et la dorsale. A l'Est du cap, la baie de Mohammedia est caractérisée par des pentes douces.

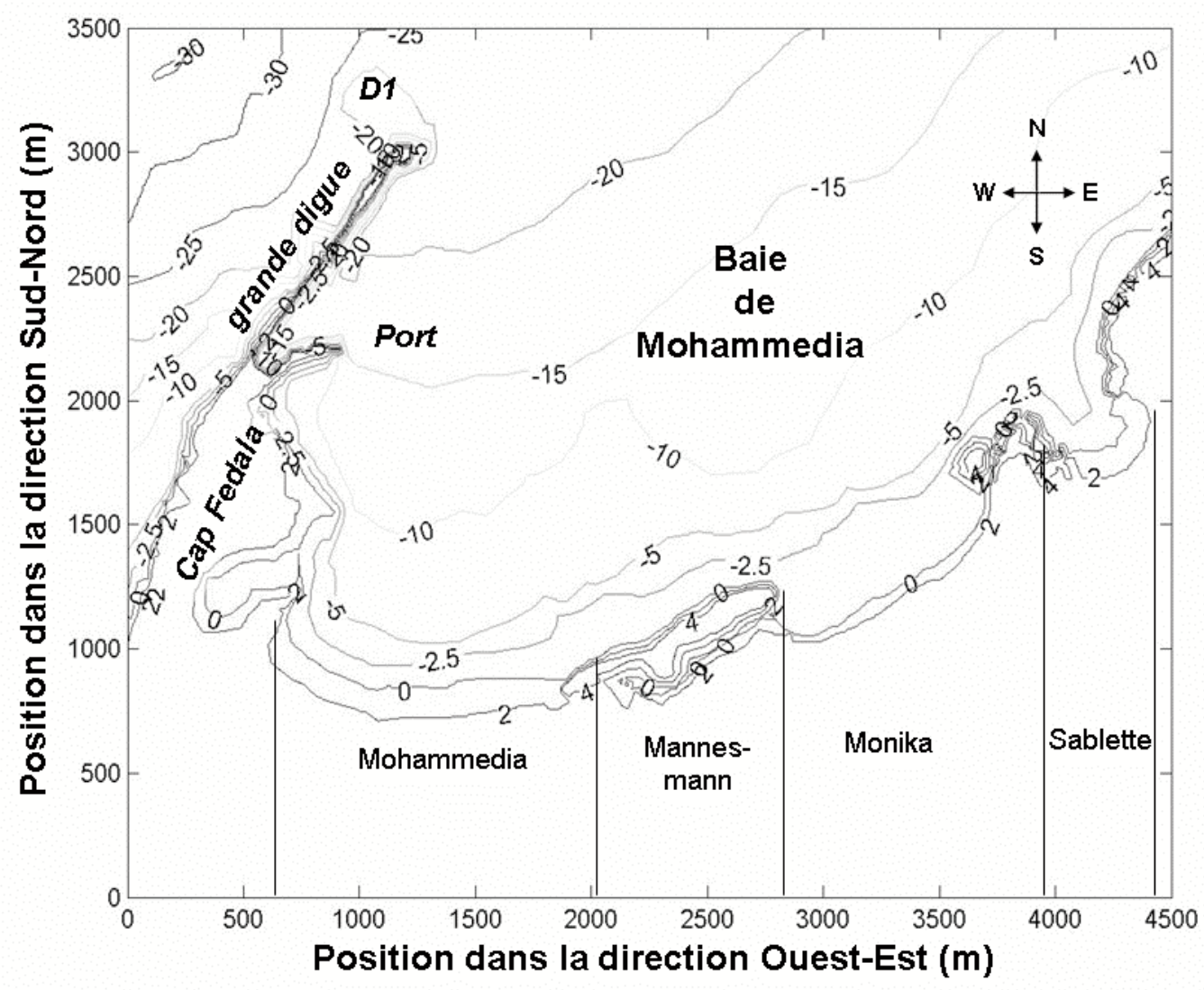

Figure 1: Bathymétrie du domaine d'étude

Cette étude fait suite à des travaux précédents réalisés sur ce littoral (Idrissi et $\mathrm{al}^{1}$ ) et qui ont montré un recul du trait de côte dans la zone de Monika et une avancée dans la zone de Mohammedia. La précédente étude avait montré que les mesures annuelles surestimaient fortement la tendance d'avancée ou de recul, comparé à une approche basée sur des données de trait de côte sur plusieurs décennies. Cependant, la méthode de calcul utilisée est basée sur le calcul du flux sédimentaire déduit de la houle au large qui ne tient pas compte de la bathymétrie locale et de l'influence des infrastructures côtière, en particulier la grande digue du port.

Afin de mieux comprendre le fonctionnement de la dynamique sédimentaire dans la baie de Mohammedia, on a utilisé une approche constituée d'une approche descriptive suivie et complétée par une approche numérique dans le but d'étudier l'évolution à long terme du trait de côte. 


\section{Approche descriptive}

\subsection{Etude granulométrique et minéralogique}

Les analyses granulométrique et minéralogique des sables prélevés le long de ce littoral pendant la période 2001-2002 (figure 2) ont montré que les plages du secteur Est sont formées de sables moyens à grossiers avec des minéraux lourds résistants. A l'Ouest, la plage de Mohammedia est constituée essentiellement de sédiments fins dont des minéraux lourds fragiles.

L'Est, soumis à l'action directe de la houle du large, subit un vannage des sables fins vers le large alors qu'à l'Ouest, protégée par la digue, la plage reçoit des vagues de faible énergie qui n'ont aucun effet érosif. La forte présence d'Augite parmi les minéraux lourds révèle également les rôles de l'oued Nfifikh (Hourimeche ${ }^{2}$ ) et d'une dérive littorale dans la dynamique sédimentaire.

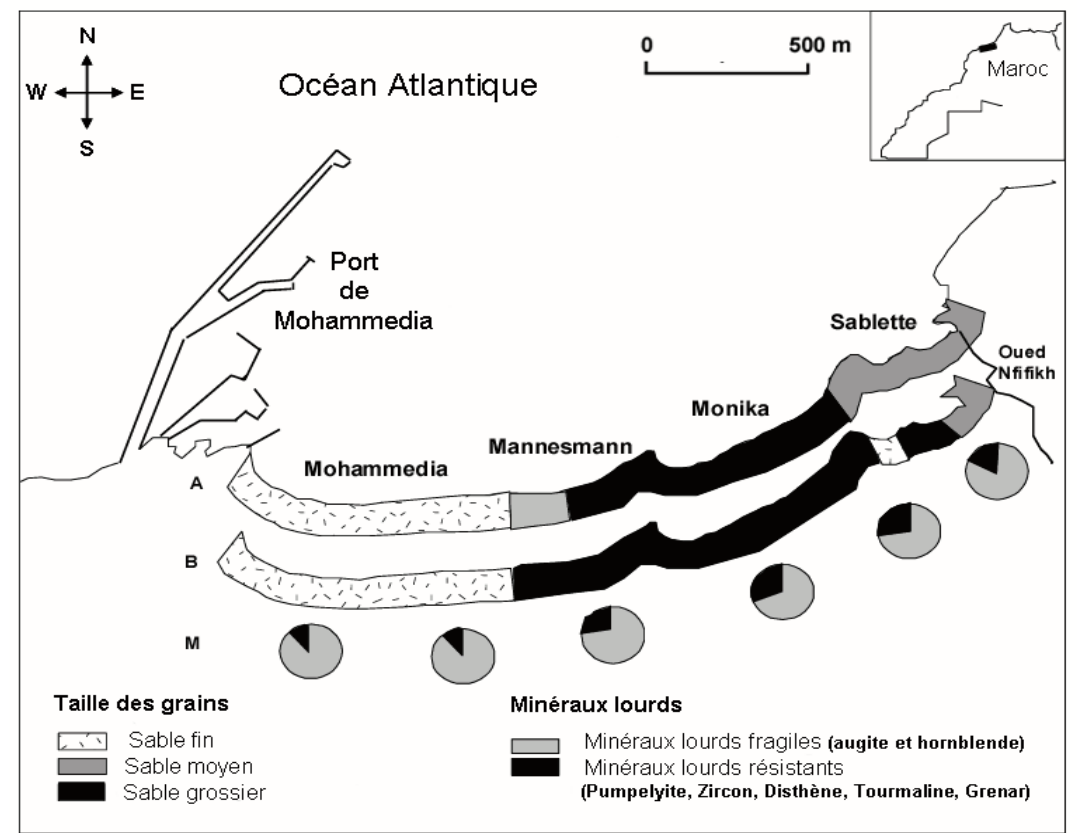

Figure 2 : Distribution granulométrique et numérique du sable du littoral de Mohammedia A: période hivernale $\quad$ B: période estivale $\quad$ M: minéraux lourds

\subsection{Evolution du trait de côte}

L'étude de l'évolution du littoral a été réalisée à partir de l'analyse des photographies aériennes de la zone de Mohammedia, des années 1969, 1986, 1997 et 2003 (figure 3). La zone se subdivise en deux secteurs. Un premier, à l'Est, est en érosion avec un recul du trait de côte estimé à $51 \mathrm{~m}$ pendant 34 ans, soit un taux de démaigrissement compris entre 0,6 et 1,6 m/an.

Le secteur Ouest est en revanche en accrétion : les plages de ce secteur subissent un engraissement, matérialisé par un ensablement régulier du port de Mohammedia. Le taux d'engraissement de la plage de Mohammedia est de $38 \mathrm{~m}$ sur 34 ans, soit environ $1 \mathrm{~m} / \mathrm{an}$. 


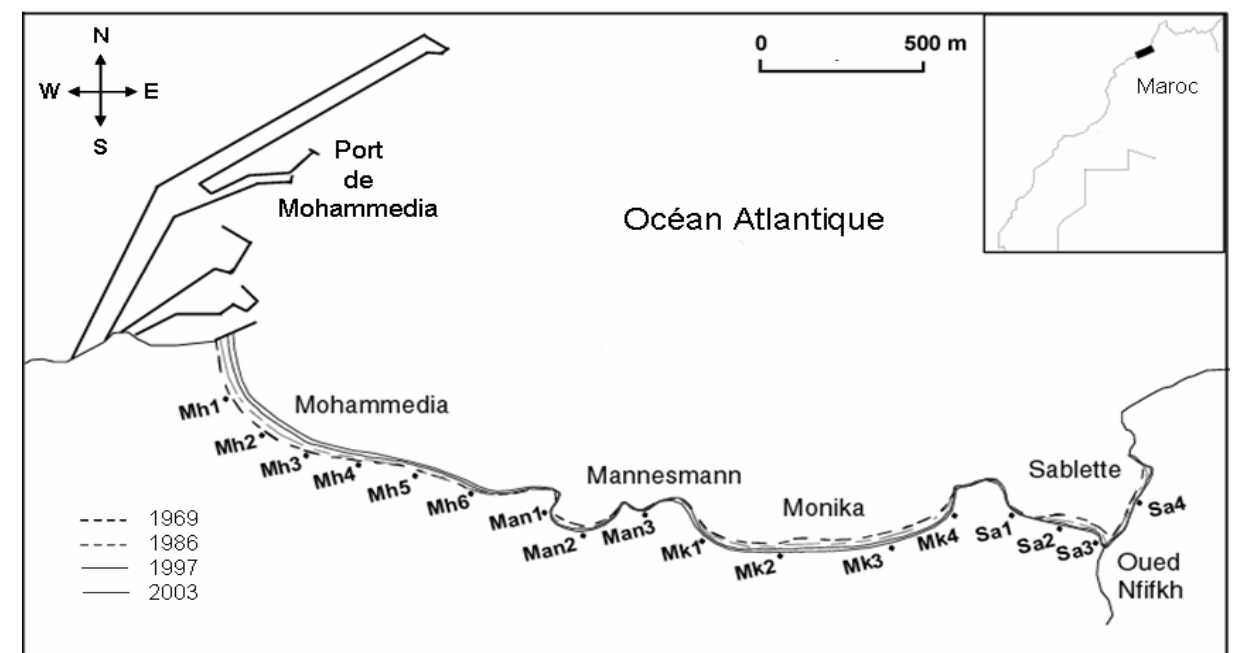

Figure 3: évolution du trait de côte du littoral de Mohammedia (Maroc) entre 1969 et 2003

\section{Approche Numérique}

Dans cette approche visant à estimer les taux d'engraissement et de démaigrissement, on propose une démarche basée sur des outils de calcul numérique. On utilise un modèle de propagation de la houle pour localiser les zones de convergence, et un modèle de calcul de l'évolution du trait de côte pour estimer le débit sédimentaire. Le modèle de houle prend en compte les effets combinés de la diffraction et de la réfraction, REFDIF $\left(\right.$ Kirby $\left.^{3}, 1986\right)$. Le critère de déferlement utilisé par le modèle est la relation qui lie la hauteur de houle et la profondeur:

$$
H_{B}=\gamma \cdot d_{B}
$$

$H_{B}$ est la hauteur de houle au déferlement, $d_{B}$ est la profondeur au déferlement et $\gamma$ est le coefficient de déferlement pris égal à 0,78 .

Le modèle de calcul de l'évolution du trait de côte résout l'équation (Horikawa ${ }^{4}$, 1988) :

$$
\frac{\partial y}{\partial t}=-\frac{1}{D}\left(\frac{\partial Q}{\partial x}\right)
$$

où $Q$ est le débit sédimentaire, $D$ est la profondeur de fermeture, $y$ est la position perpendiculaire à la côte, $x$ est la position le long de la côte et $t$ est le paramètre temps. Le débit $Q$ est calculé par la formule du CERC (Krauss et Harikai ${ }^{5}, 1983$ ):

$$
Q=H_{B}^{2} C_{g B}\left(K_{1}^{\prime} \cdot \sin \left(2 \cdot \alpha_{B P}\right)-K_{2}^{\prime} \cos \left(\alpha_{B P}\right) \frac{\partial H_{B}}{\partial x}\right)
$$

avec $\alpha_{\mathrm{BP}}$ l'incidence de la houle au déferlement par rapport à la plage, $\mathrm{C}_{\mathrm{gB}}$ la vitesse de groupe au déferlement. $x, y$ sont les coordonnées cartésiennes. $\mathrm{K}_{1}$ et $\mathrm{K}_{2}$ sont deux constantes empiriques.

Deux modèles sont ainsi couplés. Le calcul numérique de la houle est utilisé pour obtenir les caractéristiques au déferlement et pour le calcul de l'évolution du trait de côte. 


\subsection{Conditions de houle}

L'étude de la houle depuis vingt ans (1983-2003), montre que la direction de propagation dominante est NW. La houle significative a pour caractéristiques une période de $15 \mathrm{~s}$ en hiver et de $7 \mathrm{~s}$ en été, pour respectivement une hauteur de $3,4 \mathrm{~m}$ et de $2 \mathrm{~m}$. Le régime des marées est semi diurne. Le marnage moyen est de $2,2 \mathrm{~m}$ avec un maximum à $3,5 \mathrm{~m}$ et un minimum à $0,9 \mathrm{~m}$.

La modélisation de la propagation de la houle est menée en utilisant la bathymétrie de 1993 illustrée sur la figure 2. Les résultats sont présentés, pour une houle significative hivernale, en figure 4. La zone s'étendant du port à la partie Est de Mannesmann est particulièrement abritée. Puis l'agitation devient de plus en plus importante en s'éloignant vers l'Est. Ainsi, au niveau des côtes de Monika et de Sablette, l'amplitude de la houle, dans les zones de focalisation, est environ $30 \%$ plus importante qu'au large. Pour des houles hivernales et estivales, les données au déferlement (figures 5.a et 5.b) montrent une diminution quasi linéaire de la hauteur de houle depuis la plage de Sablette où la digue n'a presque pas d'influence, jusqu'à la plage de Mohammedia où l'agitation est quasiment nulle. Dans la baie, la houle est soumise essentiellement à la déformation par diffraction. On observe sur la figure 6 ce phénomène dont l'effet est prolongé jusqu'au platier rocheux de Mannesmann. Cela entraîne la création d'un courant qui ramène les sédiments de la plage Monika vers la plage Mohammedia et qui est renforcé par le courant de dérive littorale, circulant du Nord vers le Sud le long de la frange côtière. De ce fait, le secteur Ouest du littoral de Mohammedia, protégé par la jetée principale, se comporte comme une pompe aspirant en permanence les sables du secteur Est. L'élargissement et la surélévation de la plage Mohammedia sont en relation avec la présence de l'épi transversal qui protège le port de Mohammedia et qui oblige le courant de dérive littorale à déposer une partie de sa charge sédimentaire au niveau de ce secteur Ouest. Les traceurs minéralogiques utilisés dans la partie descriptive confirment cette hypothèse d'un transport suivant la direction Est-Ouest.

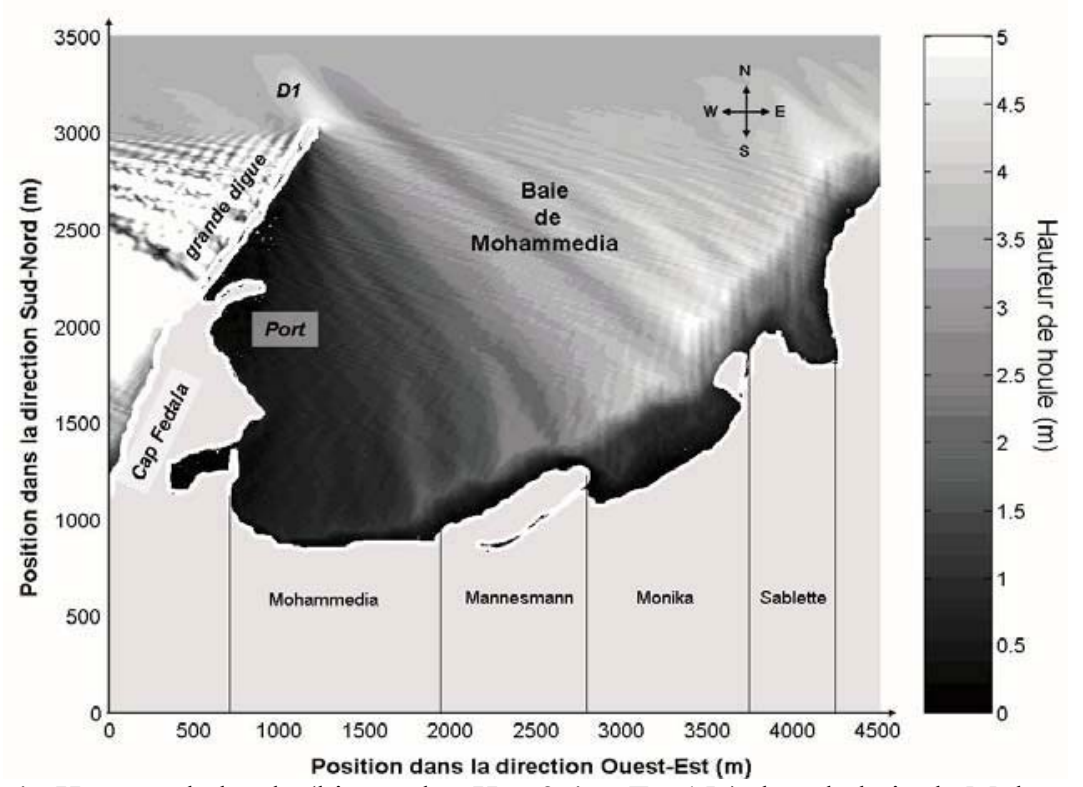

Figure 4 : Hauteur de houle (hivernale : $\mathrm{Hs}=3.4 \mathrm{~m}, \mathrm{Ts}=15 \mathrm{~s}$ ) dans la baie de Mohammedia 

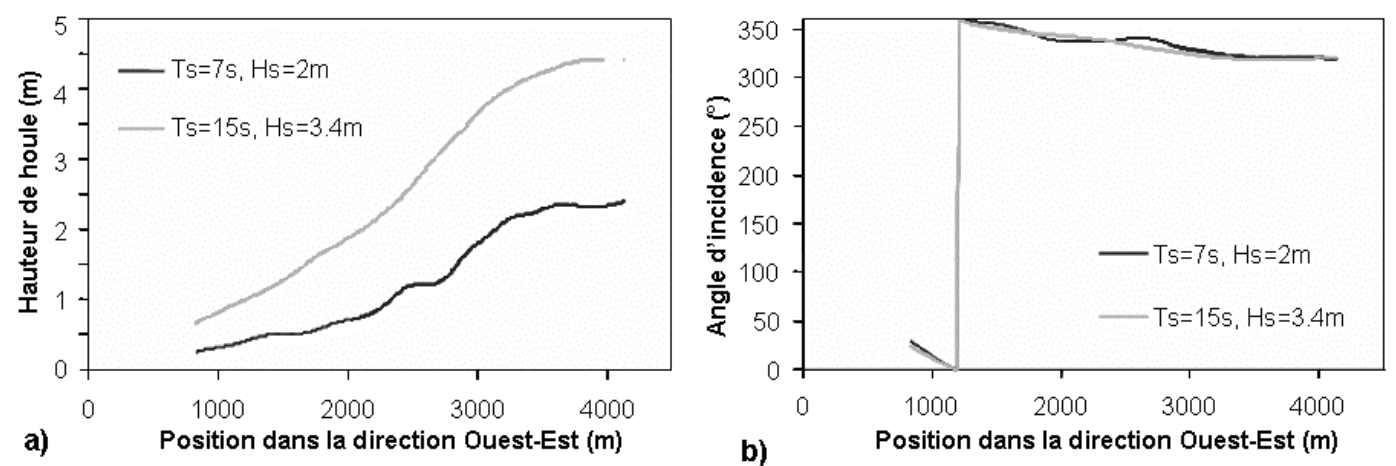

Figure 5 : Hauteurs (a) et incidence des vagues au déferlement (b) le long de la côte

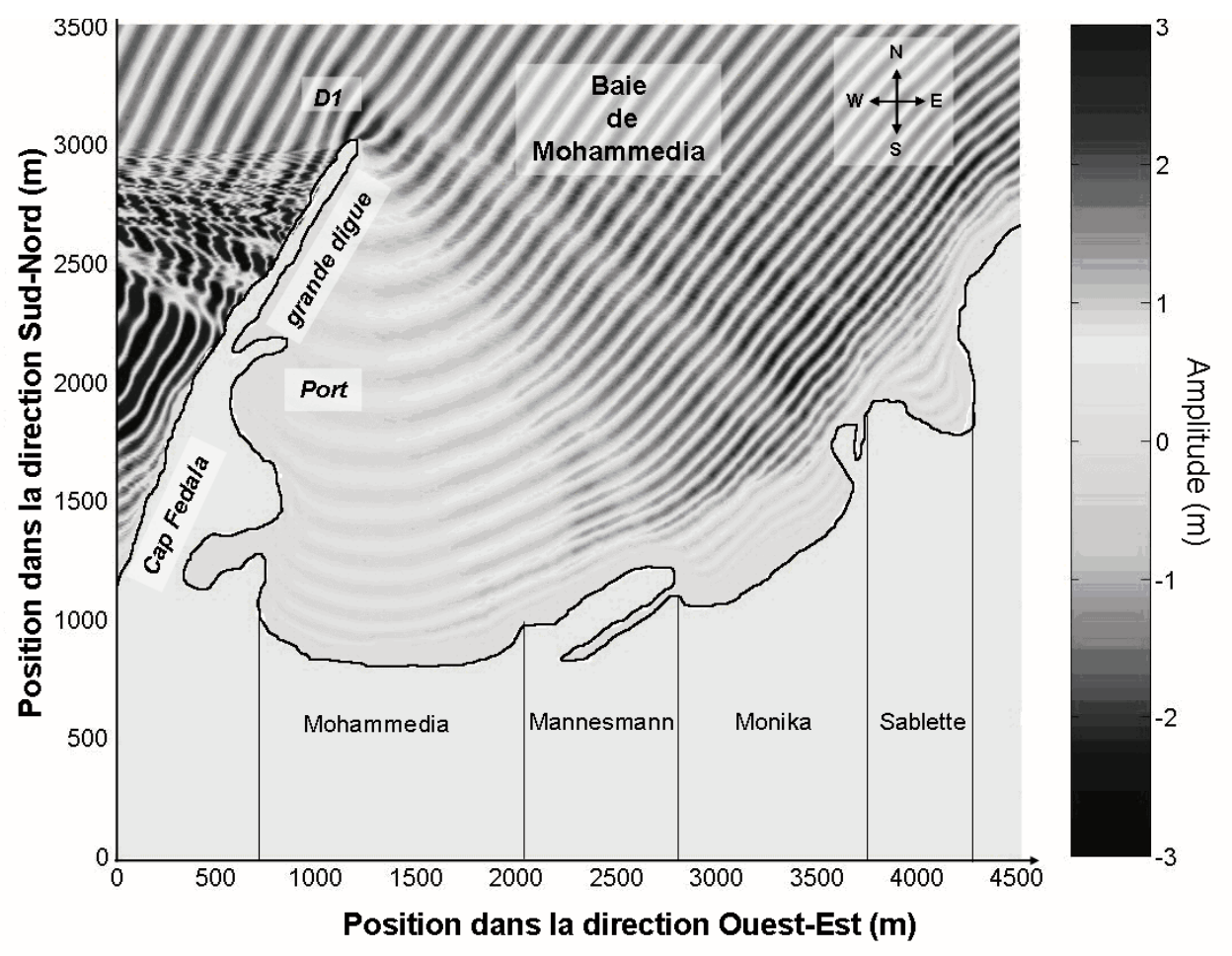

Figure 6 : visualisation des lignes de crête

\subsection{Calcul de l'évolution du trait de côte}

L'équation du flux (3) dépend des constantes empiriques $\mathrm{K}_{1}$ et $\mathrm{K}_{2}$ qui caractérisent deux types de transport. Le premier est dû à l'incidence de la houle et le second correspond aux variations de sa hauteur le long de la côte. Ils sont déterminés par la calibration du modèle d'évolution du trait de côte (Krauss et Harikai $^{5}, 1983$ ). En utilisant les données sur le trait de côte de 1993, 1997 et 2003, on obtient par une méthode itérative les valeurs suivantes :

$$
\mathrm{K}_{1}=5.5510^{-5} \quad \text { et } \quad \mathrm{K}_{2}=830.10^{-5}
$$

Sur la figure 7 , on présente l'évolution du trait de côte en fonction de la distance le long de la côte. On observe deux secteurs bien distincts: le secteur Ouest en accrétion et le secteur est en érosion. Le point d'inflexion se trouve sur la plage de Monika (Figure 8). Ce modèle, grâce aux données fournies par 
l'approche descriptive, permet de donner une évolution à long terme réaliste. Il montre que si aucun aménagement spécifique n'est réalisé, la partie Est va continuer à subir un phénomène d'érosion important et la partie Ouest s'ensablera à un rythme rapide.

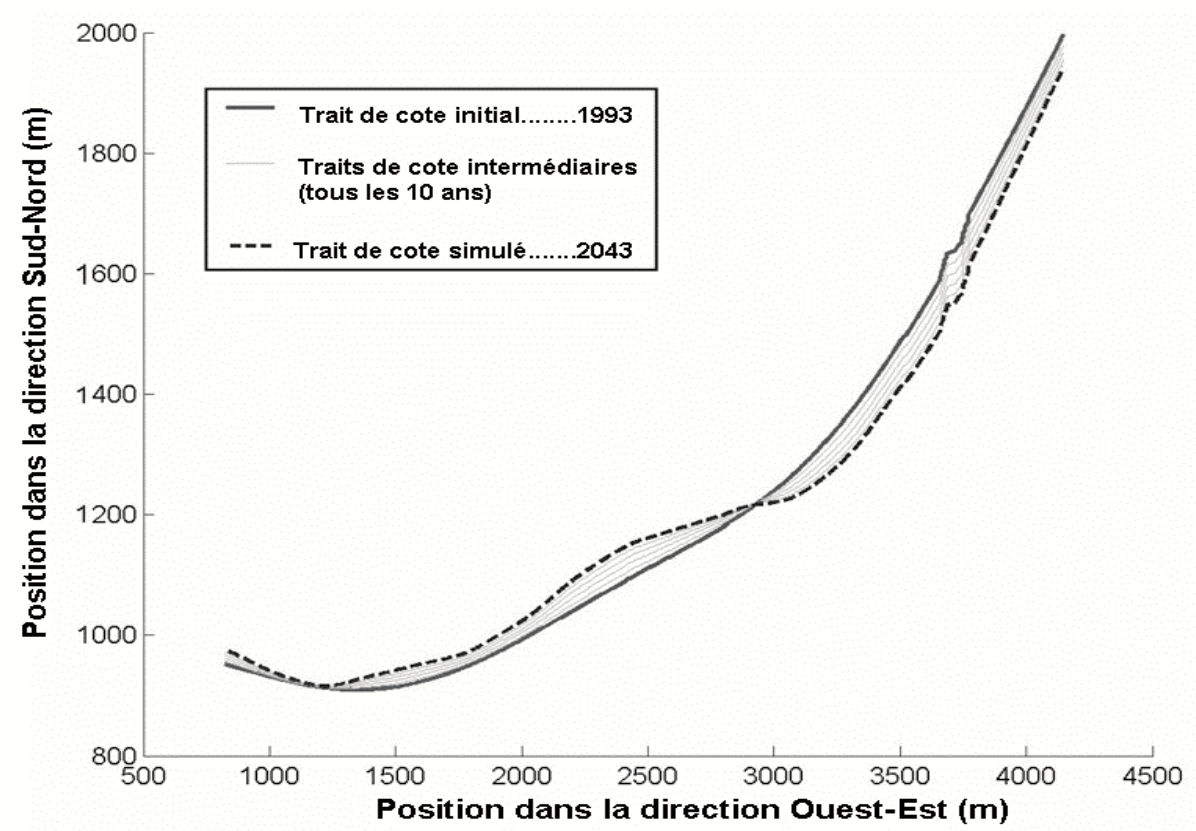

Figure 7 : Evolution du trait de côte de la baie de Mohammedia

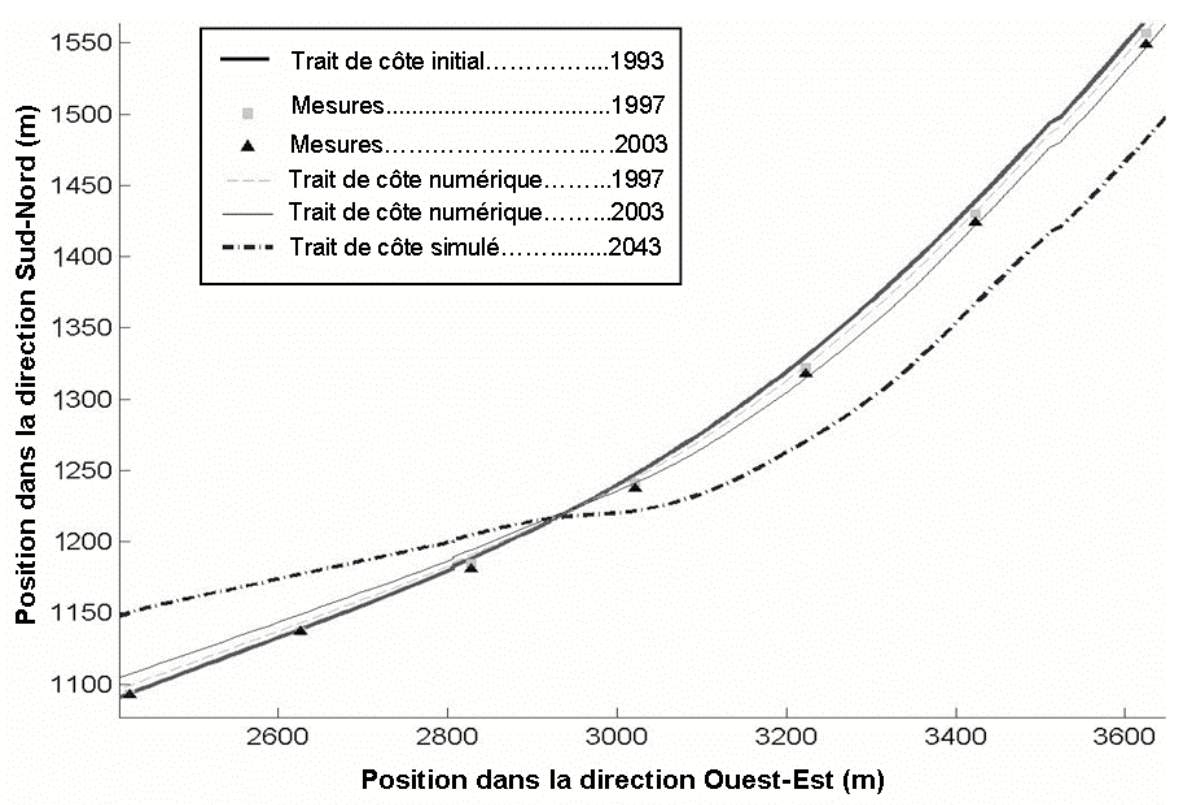

Figure 8 : Calage du modèle d'évolution du trait de côte

\section{Conclusion}

Les analyses sédimentologique et minéralogique permettent de localiser de façon qualitative les zones d'érosion et d'accrétion et donnent de ce fait des 
informations intéressantes sur la dynamique sédimentaire. Cependant, l'évolution $\mathrm{du}$ trait de côte ne peut pas être quantifiée. L'utilisation d'un modèle de propagation de vagues permet d'accéder à la prédiction des zones de convergences de la houle et par conséquent aux secteurs du littoral en érosion. La localisation des points de déferlement et le calcul des gradients longitudinaux du débit sédimentaire permettent de calculer le profil du trait de côte en calibrant les modèles sur les données disponibles. L'évolution future du trait de côte peut alors être calculée, en supposant les paramètres hydrodynamiques et morphologiques invariants.

Sur le site d'étude choisi, on a mis en évidence deux secteurs morpho sédimentaires, un exposé à la houle du large et en érosion et un autre protégé par la digue et en accrétion. Leur évolution morphodynamique est en relation avec les facteurs naturels et les travaux d'aménagement réalisés au niveau de ce littoral. L'étude de l'effet de la construction de la digue se manifeste par un déplacement des secteurs en érosion vers l'Est et le transport s'accélère vers le port par l'effet de diffraction. La comparaison des volumes de sables dragués au niveau du port de Mohammedia entre 1969 et 1992, montre que la quantité annuelle moyenne de sables dragués après la réalisation de la grande jetée portuaire est de quatre fois supérieure que celle draguée avant les travaux de la réalisation de la grande jetée portuaire (SOGREAH et $\mathrm{LPEE}^{6}$, 1994). Ceci confirme donc le rôle de l'implantation des ouvrages côtiers sur l'augmentation régulière de la morphodynamique au niveau du domaine littoral de Mohammedia.

\section{$\underline{\text { Remerciement }}$}

Ce travail a été mené dans le cadre de l'action intégrée avec le Maroc, MA/03/77 et les auteurs remercient ce programme pour son soutien financier.

\section{Bibliographie}

1 Horikawa K., (1988). Nearshore dynamics and coastal processes: theory, measurements and predictive models.

2 Hourimeche A., (1999). Etude sédimentologique des formations littorales plioquaternaires et des dépôts côtiers actuels de la région d'Essaouira - comparaison avec la région de Casablanca-. Thèse Doctorat d'Etat. Fac. Sc. Ben M'Sik, Casablanca ; $271 \mathrm{p}$.

3 Idrissi M., Hourimeche A., Rey V. et Chagdali M., (2004). Impact des installations côtières sur l'évolution du littoral de Mohammedia (Maroc). VIIlèmes Journées Nationales Génie Côtier - Génie Civil, Tome 4, pp 45-53, Compiègne, France.

4 Kirby J. T., (1986). Rational approximations in the parabolic equation method for water waves. Coastal Engineering, 10, pp. 355-378.

5 Krauss N. C. et Harikai S., (1983). Numerical model at the shoreline change at Oarai Beach, Coastal Engineering, Vol 7, pp. 1-28.

6 SOGREAH, (1994). Port de Mohammedia, Etude de l'impact du développement du port sur l'environnement. Dossier 51-2193, SOGREAH et LPEE (MAROC), Octobre 1994 